\title{
SCTP as mobility protocol for enhancing Internet on the train
}

Daan Pareit, Ingrid Moerman, Piet Demeester

Ghent University - IBBT

Department of Information Technology

IBCN Research group

Gaston Crommenlaan 8 box 201

B-9050 Ghent, Belgium

Email: $\{$ firstname.lastname $\} @$ intec.ugent.be

\author{
Wim Torfs, Peter De Cleyn, Chris Blondia \\ University of Antwerp - IBBT \\ Department of Mathematics and Computer Science \\ PATS Research group \\ Middelheimlaan 1 \\ B-2000 Antwerp, Belgium \\ Email: \{firstname.lastname\}@ua.ac.be
}

Index Terms-sctp, railway, train, mobility

Abstract-Internet-on-the-train is a rising concept in the last few years. Several trials in different countries have proved the feasibility of offering Internet access to train commuters, but none of them combines broadband access, scalability, seamless handover and quality of service guarantees in one solution. In this paper, we look at the features of SCTP and its extensions which could overcome these shortcomings and which were successfully demonstrated.

\section{INTRODUCTION}

Several wireless technologies can be used for offering broadband Internet access on trains. A train operator can have a preferred main wireless access network, but vertical handovers (switching from one technology to another) to one or more alternative gap filler wireless access networks will nevertheless be unavoidable.

For satellite networks this is due to the lack of Line of Sight in dense urban regions or vegetation, tunnels etc., whilst an incomplete national coverage for trackside communications also requires other technologies to serve as gap fillers. Interworking between heterogeneous wireless technologies is thus inevitable.

One of our activities within the IBBT GBO Tr@ins project led to the design of a Mobility Management Module, to be installed in all trains as well as in a central Network Operations Center of the train operator (see Figure 1). This Mobility Management Module covers both the Handover Management and the Quality of Service (QoS) support for various applications for passengers and crew. Furthermore, those components are orchestrated by a Policy Decision Function (PDF) which notifies them of an imminent handover, based on link conditions, location information etc (see Figure 2).

As for the Mobility Management Module, we implemented two different mobility protocols. The first one is based on Mobile IP (MIP), while the second one is founded on the Mobile Multi-Path Stream Control Transmission Protocol (MMPSCTP).

We successfully investigated and demonstrated the possibility for using (MMP)-SCTP as the mobility and tunneling

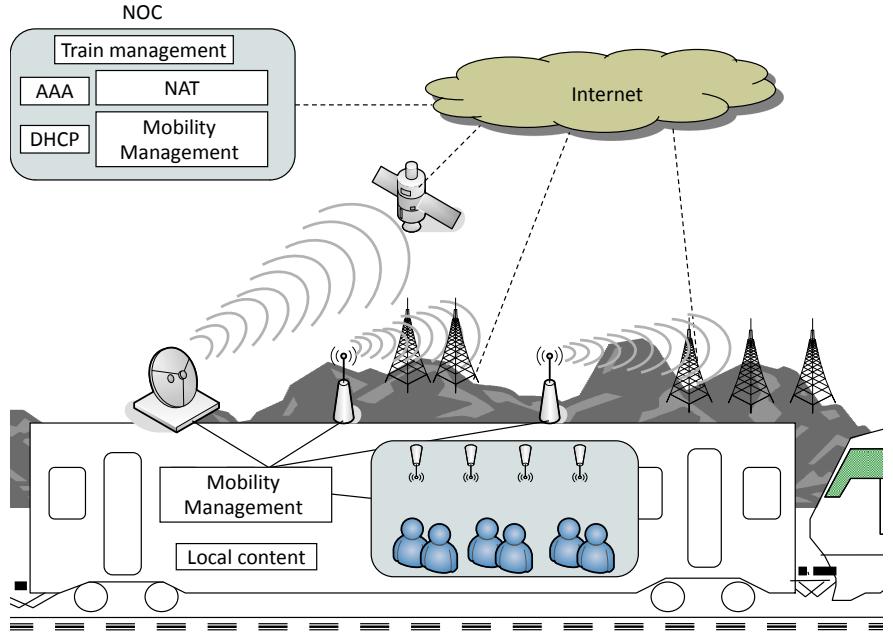

Fig. 1. Tr@ins general architecture

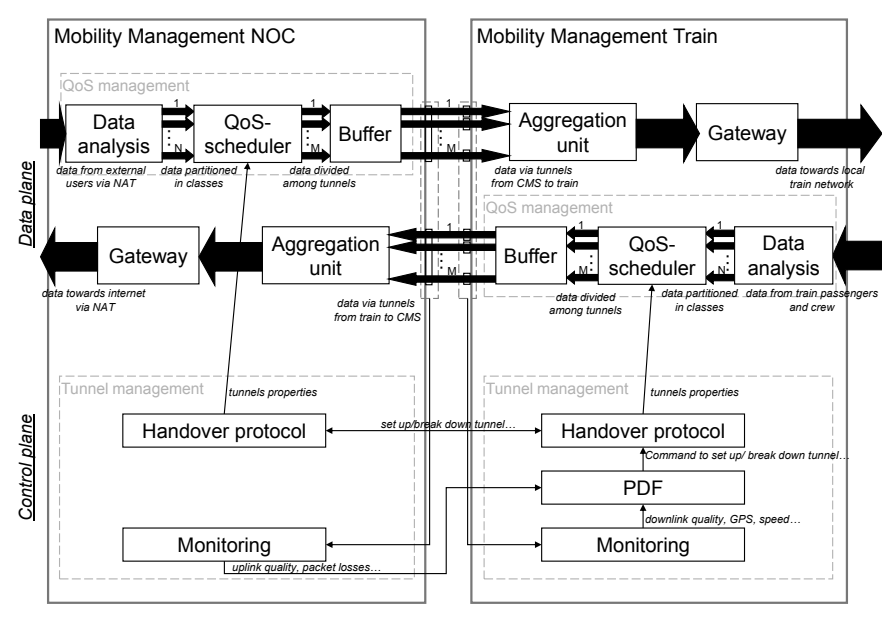

Fig. 2. Mobility management architecture 


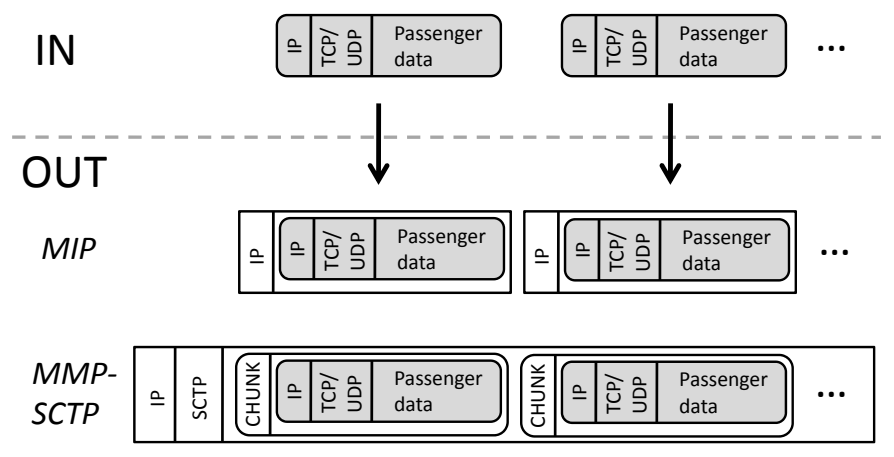

Fig. 3. MIP vs SCTP encapsulation of passenger packets

protocol between a train and a Network Operations Center (NOC) on the main land. The latter serves as gateway, firewall and NAT (when using IPv4) for the trains toward the Internet.

In [1] we measured vertical handovers between available mobile networks of incumbent operators, indicating the need for a efficient mobility protocol. We developed an IP network architecture with QoS support in a transparent way: passengers on the train should not be aware of the handovers and should enjoy an uninterrupted seamless multimedia network experience.

In this paper we highlight the advantages SCTP has in a train scenario. First some general information about SCTP is given. Next we discuss packet aggregation with SCTP in a wireless LAN. In the following section the multihoming feature of SCTP is discussed. Finally the reliability aspect is described.

\section{SCTP BASICS}

SCTP (Stream Control Transport Protocol) [2] is a reliable transport protocol on top of an potential unreliable packet service like IP. Selective acknowledgments (SACKs) are used for confirmation and retransmission of SCTP chunks (a packet consists of one or multiple chunks) when packet loss is detected, e.g. during a handover.

The use of a transport protocol as a tunneling protocol between a train and the NOC of the train operator could appear strange at first sight, but previous research [3], [4], [5] did show that SCTP's features are suitable for use in situations with heterogeneous access technologies.

We implemented the SCTP protocol in $\mathrm{C}++$ with the Click Modular Router [6].

\section{Packet Aggregation}

SCTP provides chunk bundling. Multiple chunks are put into the same SCTP packet. In [1] we showed that the overhead at layer 3 of the OSI protocol stack is much higher for MMP-SCTP in comparison with the use of Mobile IP (see Figure 3 ). This does however not necessarily translate in a lower throughput at layer 2 .

We can prove this by examining the theoretical achieved throughput in a WLAN for MIP versus MMP-SCTP. We consider a IEEE 802.11a WLAN with a MTU of 1500 bytes,
TABLE I

THROUGHPUT IN WLAN FOR MMP-SCTP VS MIP.

\begin{tabular}{|c|c|c|c|c|}
\hline & \multicolumn{2}{|c|}{ CSMA/CA (Mbps) } & \multicolumn{2}{|c|}{ RTS/CTS (Mbps) } \\
\hline & mSCTP & MIP & mSCTP & MIP \\
\hline 50 & 23,5 & 3,6 & 19,7 & 2,1 \\
\hline 100 & 26,4 & 6,8 & 22,1 & 4,0 \\
\hline 150 & 27,0 & 9,5 & 22,5 & 5,8 \\
\hline 200 & 27,4 & 11,9 & 22,7 & 7,5 \\
\hline 250 & 28,2 & 14,1 & 23,5 & 9,0 \\
\hline 300 & 27,7 & 16,4 & 23,0 & 10,6 \\
\hline 350 & 29,9 & 18,2 & 25,1 & 12,0 \\
\hline 400 & 28,0 & 19,8 & 23,2 & 13,2 \\
\hline 450 & 29,4 & 21,2 & 24,7 & 14,4 \\
\hline 500 & 25,7 & 22,5 & 20,9 & 15,5 \\
\hline 550 & 26,9 & 23,7 & 22,1 & 16,5 \\
\hline 600 & 28,3 & 24,7 & 23,4 & 17,5 \\
\hline 650 & 29,3 & 26,3 & 24,4 & 18,7 \\
\hline 700 & 30,2 & 27,2 & 25,3 & 19,6 \\
\hline 750 & 22,1 & 28,0 & 17,5 & 20,4 \\
\hline 800 & 22,9 & 28,8 & 18,3 & 21,2 \\
\hline 850 & 23,7 & 29,6 & 19,0 & 21,9 \\
\hline 900 & 24,4 & 30,3 & 19,7 & 22,6 \\
\hline 950 & 25,1 & 30,9 & 20,3 & 23,3 \\
\hline 1000 & 26,1 & 32,0 & 21,2 & 24,2 \\
\hline 1050 & 26,7 & 32,6 & 21,8 & 24,9 \\
\hline 1100 & 27,2 & 33,1 & 22,3 & 25,4 \\
\hline 1150 & 27,8 & 33,6 & 22,9 & 26,0 \\
\hline 1200 & 28,3 & 34,0 & 23,4 & 26,5 \\
\hline 1250 & 28,8 & 34,5 & 23,9 & 27,0 \\
\hline 1300 & 29,3 & 34,9 & 24,4 & 27,5 \\
\hline 1350 & 30,1 & 35,8 & 25,1 & 28,3 \\
\hline 1400 & 30,5 & 36,1 & 25,6 & 28,7 \\
\hline
\end{tabular}
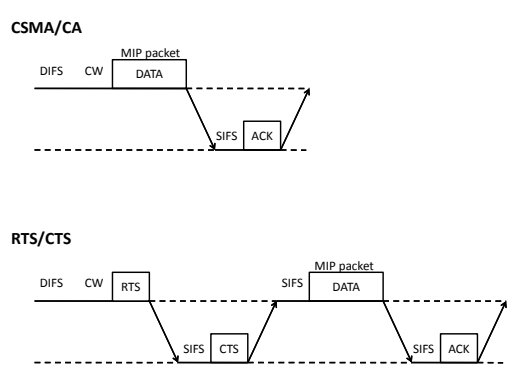

Fig. 4. MIP in WLAN

a contention window of 0 time slots, a physical data rate of $54 \mathrm{Mbps}$ and a negligible propagation delay.

For MIP the medium access with CSMA/CA and with RTS/CTS is shown in Figure 4.

Based upon those schemes we can calculate the time needed to send one passenger packet. The ratio of the number of bits in this passenger packet to the total time needed to send the encapsulating MIP packet gives us the useful bit rate at which passenger packets are sent. For both CSMA/CA and RTS/CTS those figures are shown in Table I.

For MMP-SCTP we can likewise create access schemes, though somewhat more complicated as we need to consider chunk aggregation and a SACK chunk traveling in the opposite direction. For CSMA/CA and for RTS/CTS the scheme is shown in 5.

Again, we can calculate the total time needed for a sequence of each scheme to pass. The ratio of the total amount of bits 

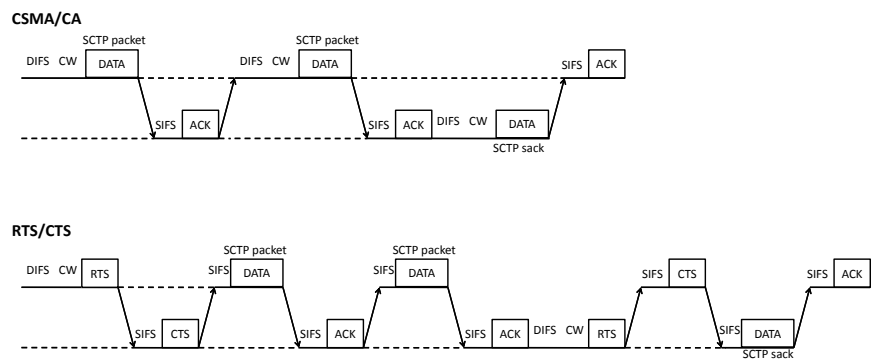

Fig. 5. MMP-SCTP in WLAN

of passenger data packets (see Figure 3) to this calculated time gives us the useful bit rate at which passenger packets are sent. For both CSMA/CA and RTS/CTS those figures are shown in Table I.

When comparing the L2 throughput for CSMA/CA and RTS/CTS in Table I, we see than we obtain much higher throughputs for MMP-SCTP when passenger packets are small. When passenger packets become large, MIP achieves a higher throughput. This is to be expected due to the MMPSCTP chunk aggregation and for the larger the L2 packets are, the higher the L2 throughput will be. This is because each L2 packet needs to wait a certain amount of time before it can be sent, and each L2 packet involves the transmission of a L2 ACK for acknowledgment. If one is able to send large packets, the relative amount of waiting time and overhead per byte of the L2 packet decreases. As a consequence the average time to send a byte decreases and thus the throughput increases. When passenger packets become too large to be aggregated, we see the effect of the higher L3 overhead for MMP-SCTP [1]. Now for each passenger one SCTP or MIP packet is sent out (instead of multiple passenger packets per SCTP packet for small passenger packets). And as the SCTP L3 overhead is higher than the MIP L3 overhead, we obtain a higher throughput for passenger packets with the MIP protocol. The results are also visualized in Figure 6 and Figure 7.

These findings are especially important when considering VoIP traffic. As packets are relatively small (e.g. less than 200 bytes), the throughput in a WLAN with Mobile IP will be low. This results in bad voice quality for multiple simultaneous calls or in a limited number of supported VoIP calls (when applying call admission and guaranteeing a certain bandwidth per call). Should one use SCTP's chunk aggregation capabilities, we have a significant higher throughput, supporting more calls.

There however still exists a problem with the aforementioned solution. For a multihomed endpoint SCTP [2] specifies that fragmentation should be done according to the smallest MTU of all available paths, the so called association path MTU (PMTU). In this case, when the train has a link with a smaller MTU than its WLAN link, the SCTP packet size will be suboptimal, resulting in a lower throughput than maximum achievable. Therefore we did not implement an association path MTU, but we fragment on each link according to its own

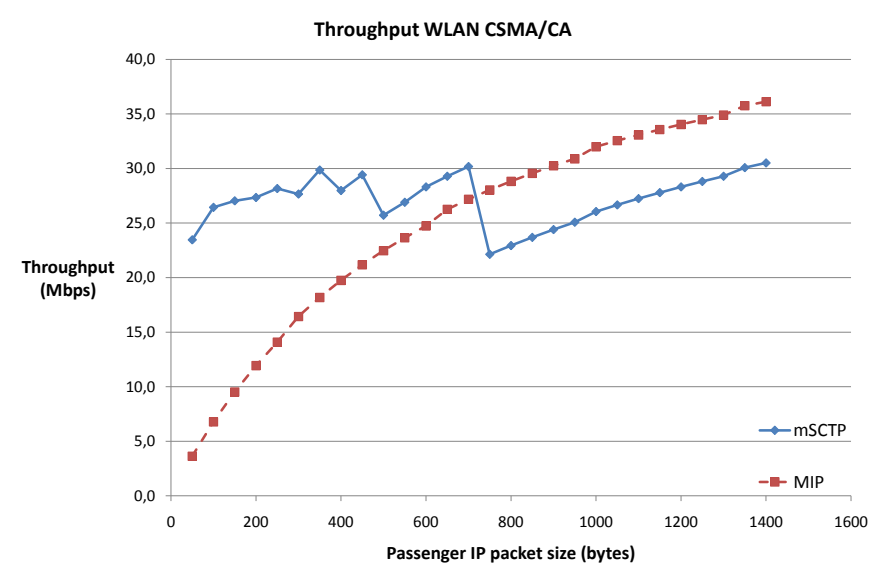

Fig. 6. Throughput WLAN with CSMA/CA: MMP-SCTP vs MIP

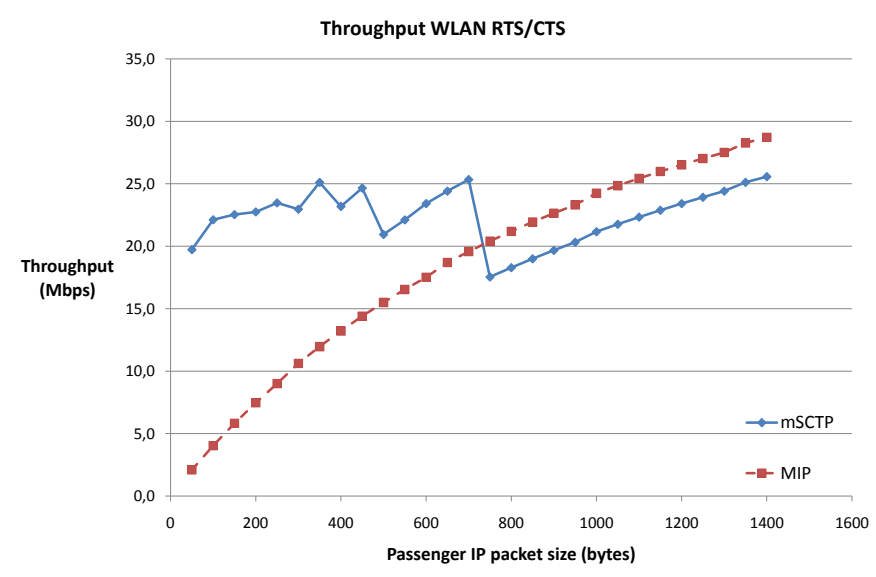

Fig. 7. Throughput WLAN with RTS/CTS: MMP-SCTP vs MIP

path MTU. This way, SCTP packets can have a maximum packet size. If retransmission of a SCTP fragmented packet on another link occurs and this link has a lower MTU, than we rely on the IP fragmentation for fragmenting this packet in order to be able to send it on the link with the smaller MTU.

We end this section with some remarks. Firstly, in order to simplify calculations, we supposed a unidirectional data connection. An approximation of a bi-directional data connection could easily be achieved by supposing a higher contention window which will yield the same conclusions. Secondly, note that for MMP-SCTP no passenger packets are fragmented before placing them into a SCTP packet. This also explains the non-smooth graph for MMP-SCTP. If fragmentation would be done, it could even slightly improve the performance for MMP-SCTP as all SCTP packets would be completely filled to the maximum MTU, reducing the relative amount of L2 overhead per passenger packet. Thirdly, for MMP-SCTP, SACK chunks are here considered to be sent back in a separate SCTP packet. With bidirectional data traffic, the SACK chunk can piggyback with other data, which will eliminate the need for this small SCTP packet (with solely a SACK chunk aboard) to be sent and thus improving L2 throughput. 


\section{Multihoming}

Within the IETF MEXT (continuation of the former MIP6, NEMO, and MONAMI6) working group there is a lot of interest in adding multihoming to Mobile IP [7], but this has not resulted in any standard yet. SCTP however incorporates multihoming directly in its standard. While for standard SCTP the IP addresses need to be configured statically at initialization time, the ASCONF [8] extension supports dynamically changing IP-addresses during an SCTP association. For the train scenario, this means that a train will initially set up an association with the NOC, but within this association it will add and remove paths (wireless links) as it rides along its track and enters and leaves the coverage area of various supported wireless access technologies.

However, there are some drawbacks too. SCTP is multihomed, but it uses only one path at a time (the primary path). The other paths (secondary paths) within the association are merely to be considered as 'backup paths'. SCTP is developed as a transport protocol, to be used instead of e.g. TCP or UDP. If one should use multiple paths simultaneously, those paths have some IP routers in common, making them non disjoint paths. This partly justifies why not using multiple paths with SCTP for load balancing. For our train scenario however, multiple paths in SCTP always use different access technologies. The path continues along the aggregation and core network to the NOC, where paths can still have part of them in common. This will typically not pose any problems as it is the wireless access networks which cause the bottlenecks. In [9] there were some concepts suggested to use multiple paths in SCTP simultaneously. This concept was given the name Mobile MultiPath SCTP (MMP-SCTP).

Using multiple paths at the same time can (partly) solve some of the above mentioned problems with the current trials: bandwidth constraints and QoS provisioning. With load balancing we now have extra bandwidth available as we use multiple links simultaneously and we can support better QoS guarantees by mapping e.g. interactive data traffic on lowdelay links while mapping bulk data traffic to high bandwidth links. Introducing load balancing however immediately requires the need for a more complex QoS scheduler as packets will need to be classified and a link will need to be chosen where packets will be sent on.

On our testbed (see Figure 8) we successfully implemented the use of multiple simultaneous paths and built a QoS scheduler as depicted in the data plane in Figure 2. The elaborated architecture is shown in Figure 9. Packets are partitioned into 5 different QoS classes in the Classifier, by means of a data analysis. The 5 classes used are:

- Background

- Best effort

- Streaming

- Interactive

- Priority

The data analysis is done by analyzing source and destination ports of UDP or TCP packets. A number of typical

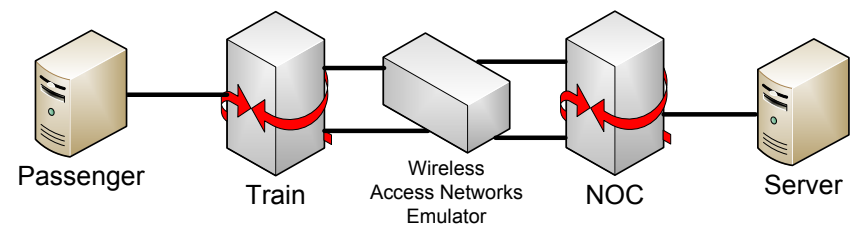

Fig. 8. Our testbed set-up for implementing and testing SCTP

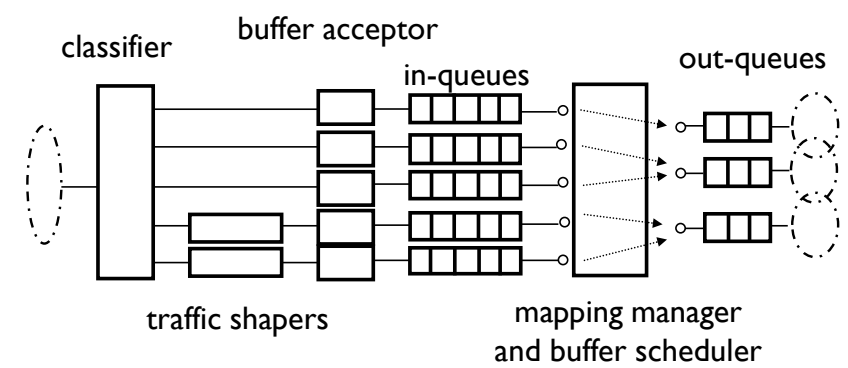

Fig. 9. QoS architecture

ports, the corresponding protocol and the QoS class they are mapped to are shown in Table II. Furthermore, passenger packets are mapped to a specific QoS class based on their IP or MAC address. This enables us to give priority to crew members, based on the addresses of their devices. After QoS classification, the passenger packets can be shaped and admission control can be applied if necessary.

According to the available wireless access networks and their characteristics we then map each class to one of those networks based on the latency and bandwidth requirements of each class. When multiple classes are mapped on the same link, schedulers will be used to make a distinction between the different classes. With our implementation we were able to show that a high priority audio stream (e.g. for train personnel) is played uninterruptedly in real-time, where this was not possible when we did not use any QoS services.

\section{Reliability}

As SCTP was developed as a transport protocol, it incorporated concepts of TCP and UDP. One of them is reliability. Received packets are acknowledged by sending SACK chunks back to the sender, indicating the correct received packets and the missing packets, based on the TSN sequence number. The possibility also exist to disable the reliable aspect [10].

TABLE II

TYPICAL PROTOCOL PORTS AND THEIR QOS CLASSIFICATION

\begin{tabular}{|l|l|l|}
\hline Port & Protocol & QoS class \\
\hline 80 & HTTP & Best effort \\
554 & RTSP & Streaming \\
2979 & H.263 video & Streaming \\
5060 & SIP & Interactive \\
5061 & TLS over TCP & Interactive \\
8080 & HTTP (with proxy server) & Best effort \\
\hline
\end{tabular}




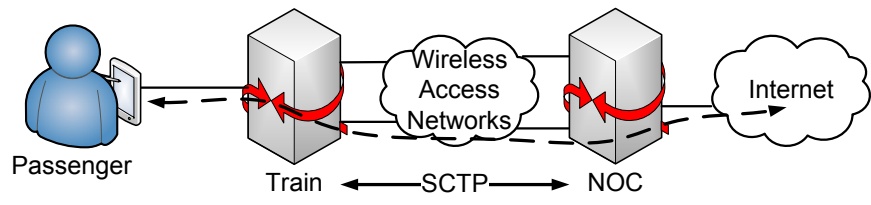

Fig. 10. (Reliable) SCTP connection between train and NOC

In the train scenario, SCTP gives us a reliable tunnel between the train and the NOC (see Figure 10). If a packet gets lost in the access network, retransmission will happen. This way, data transmission from users (passengers, crew, machines) within the train toward servers on the main land (our vice versa) will be improved. If for this data transmission an unreliable transport protocol is used (e.g. UDP), then the part of the path the packets are traveling between the train and the NOC still will be done in reliable way due the SCTP association. As the access network is the most failure prone part of the path, the SCTP reliability will greatly reduce the end-to-end packet loss. If on the hand a reliable transport protocol like TCP is used, the SCTP reliability will still improve the connection because retransmission will be done much faster if a packet is lost in the access network. This is because the packet loss will be detected by the SCTP mechanism in the NOC before it is detected by TCP at the server. This effect will be most noticeably when the latency between NOC and server endpoint is relatively large compared to the latency of the access network.

Reliability comes with a drawback however. As a sender needs to know about the correct delivery of its packets, the receiver needs to inform the sender. This requires sending acknowledgments (SACK chunks) and thus causes extra overhead. As we previously saw however that chunks can be bundled, SACKs can piggyback with other data chunks and thus minimizing their overhead.

\section{CONCLUSIONS AND FUTURE WORK}

We showed the possibility to use (MMP-)SCTP as the mobility and tunneling protocol between the train and the NOC of the train operator.

This offers features such as multihoming that are currently still being implemented for Mobile IP or features such as packet bundling and reliability that have no equivalent in currently used mobility protocols for railway vehicles. Those qualities, intrinsic to SCTP, let us obtain higher bandwidths and better QoS provisioning and thus delivering an enhanced online experience for passenger, crew and machines in a train.

We will further examine the influence of packet aggregation on throughput in other (shared) media besides WLAN and we will continue to elaborate QoS mechanisms for the train.

\section{ACKNOWLEDGMENT}

This research was carried out as part of the IBBT Tr@ins project. Daan Pareit would like to thank the IWT-Vlaanderen (Institute for the Promotion of Innovation through Science and Technology in Flanders) for financial support through his Ph.D. grant.

\section{REFERENCES}

[1] D. Pareit, N. Gheysens, T. Van Leeuwen, I. Moerman, W. Van Brussel, I. Clenjans, W. Torfs, P. De Cleyn, and C. Blondia, "Qosenabled internet-on-train network architecture: inter-working by mmpsctp versus mip," in Telecommunications, 2007. ITST '07. 7th International Conference on ITS, 2007, pp. 1-6. [Online]. Available: http://ieeexplore.ieee.org/xpls/abs_all.jsp?arnumber=4295922

[2] R. Stewart, "Stream Control Transmission Protocol," RFC 4960 (Proposed Standard), Sep. 2007. [Online]. Available: http://www.ietf.org/rfc/rfc4960.txt

[3] J. K. Song and W. Wang, "A simulation study of ip-based vertical handoff in wireless convergent networks: Research articles," Wirel. Commun. Mob. Comput., vol. 6, no. 5, pp. 629-650, August 2006. [Online]. Available: http://portal.acm.org/citation.cfm?id=1148713

[4] J. Shi, Y. Jin, W. Guo, S. Cheng, H. Huang, and D. Zhang, "Performance evaluation of sctp as a transport layer solution for wireless multi-access networks," in Wireless Communications and Networking Conference, 2004. WCNC. 2004 IEEE, vol. 1, 2004, pp. 453-458 Vol.1. [Online]. Available: http://ieeexplore.ieee.org/xpls/abs_all.jsp?arnumber=1311587

[5] S. J. Koh and S. W. Kim, "msctp for vertical handover between heterogeneous networks," 2005, pp. 28-36. [Online]. Available: http://dx.doi.org/10.1007\%2F11527725_4

[6] E. Kohler, "The click modular router," Ph.D. dissertation, Massachusetts Institute of Technology, Feb 2001. [Online]. Available: http://pdos.csail.mit.edu/papers/click:kohler-phd/thesis.pdf

[7] T. Ernst, N. Montavont, R. Wakikawa, C. Ng, and K. Kuladinithi, "Motivations and scenarios for using multiple interfaces and global addresses," IETF Internet draft, May 2008. [Online]. Available: http://tools.ietf.org/html/draft-ietf-monami6multihoming-motivation-scenario-03

[8] R. Stewart, Q. Xie, M. Tuexen, S. Maruyama, and M. Kozuka, "Stream Control Transmission Protocol (SCTP) Dynamic Address Reconfiguration," RFC 5061 (Proposed Standard), Sep. 2007. [Online]. Available: http://www.ietf.org/rfc/rfc5061.txt

[9] C. Huang and C. Tsai, "Mobile multi-path transmission using SCTP," IETF Internet draft, Oct 2006. [Online]. Available: http://www.ietf.org/internet-drafts/draft-huang-tsai-mmp-sctp-00.txt

[10] R. Stewart, M. Ramalho, Q. Xie, M. Tuexen, and P. Conrad, "Stream Control Transmission Protocol (SCTP) Partial Reliability Extension," RFC 3758 (Proposed Standard), May 2004. [Online]. Available: http://www.ietf.org/rfc/rfc3758.txt 